University of Warwick institutional repository: http://go.warwick.ac.uk/wrap This paper is made available online in accordance with publisher policies. Please scroll down to view the document itself. Please refer to the repository record for this item and our policy information available from the repository home page for further information.

To see the final version of this paper please visit the publisher's website. Access to the published version may require a subscription.

Author(s): K. JAROENSUTASINEE and G. ROWLANDS Article Title: Charged-particle orbits near a magnetic null point Year of publication: 2000 Link to published version: http://dx.doi.org/10.1017/S0022377800008461

Publisher statement: None 
(C) 2000 Cambridge University Press

\title{
Charged-particle orbits near a magnetic null point
}

\author{
K. JAROENSUTASINEE and G. ROWLANDS \\ Department of Physics, University of Warwick, Coventry CV4 7AL, UK
}

(Received 10 January 2000)

\begin{abstract}
An approximate analytical expression is obtained for the orbits of a charged particle moving in a cusp magnetic field. The particle orbits pass close to or through a region of zero magnetic field before being reflected in regions where the magnetic field is strong. Comparison with numerically evaluated orbits shows that the analytical formula is surprisingly good and captures all the main features of the particle motion. A map describing the long-time behaviour of such orbits is obtained.
\end{abstract}

The motion of charged particles in spatially varying magnetic fields has received a great amount of attention because of its relevance to plasma fusion devices, particle accelerators and astrophysics. Even in the simplest cases, the motion is complicated and is now known to be an example of chaos. One simplifying assumption, which is good when the ratio of the Larmor radius to a scale length describing the spatial variation of the magnetic field, $\varepsilon$, is small, is that the so-called adiabatic invariant $\mu$ is a constant. This immediately leads to an explanation of charged-particle containment in the Van Allen radiation belts and in magnetic mirror fusion devices. For larger values of $\varepsilon$, it has been found that the adiabatic invariant undergoes jumps $\Delta \mu$ where $\mu$ changes rapidly in just a few Larmor periods in special regions of symmetry, but otherwise $\mu$ is to all intents and purposes constant. The jumps are such that $\Delta \mu \propto \exp (-1 / \varepsilon)$. For a specific calculation of $\Delta \mu$ for a wide range of magnetic field configurations, see for example Cohen et al. (1978). In this case, the long-time behaviour of particles can be understood in terms of a map relating the values $\left(\mu_{n} \theta_{n}\right)$ before a jump to the values $\left(\mu_{n+1}, \theta_{n+1}\right)$ after a jump. Here $\theta$ is an angle specifying the Larmor phase of the particle. It is found that, to a reasonable approximation (terms of order $\exp (-2 / \varepsilon)$ being neglected), that one can write

$$
\mu_{n+1}=\mu_{n}+\Delta \mu \cos \theta_{n}
$$

where, of course, $\Delta \mu$ is a function of $\mu_{n}$. In many applications, it is sufficient to restrict attention to changes in $\mu$ that are small, so that one may linearize the value of $\mu_{n}$ about a chosen mean. Then the above equation reduces to

$$
\delta \mu_{n+1}=\delta \mu_{n}+K \cos \theta_{n},
$$

and since the original equations of motion were Hamiltonian, the equation for $\theta$ variation can be obtained by insisting that the Jacobian is unity. This gives

$$
\theta_{n+1}=\theta_{n}+\delta \mu_{n+1} \text {. }
$$

* Present address: Walaik University, Thailand. 
In the above, $K$ is a constant whose value is determined by the field configuration and energy of the particle.

The above map $(\delta \mu, \theta)$ is the Chirikov map, and is used to study the long-time behaviour of nearly adiabatic particles in spatially varying magnetic fields. For sufficiently small values of $\Delta \mu$, it is found that the particle motion is such that $\mu$ changes periodically about a constant value (superadiabatic). For larger values, the motion can become chaotic; and for sufficiently large values, the motion of the charged particle can be understood in terms of a diffusion in momentum space with diffusion coefficient proportional to $\exp (-1 / \varepsilon)$. Numerous examples of this type of behaviour have now been studied in detail, and are described in the book by Lichtenberg and Lieberman (1983).

It must be stressed that the direct numerical solution of the particle-orbit equations becomes prohibitively expensive in machine time because one has to follow the particle around its Larmor orbit, whereas it is the motion of the guiding centre that is really needed. Adiabatic and weakly non-adiabatic theory overcome this problem by essentially introducing a suitable averaging procedure to remove the fast motion associated with motion about the Larmor orbit.

However, the whole theory is totally inadequate if, during its motion, a particle can move in a region where the field strength is small or even zero. An example of such a field is the two-dimensional cusp described by the vector potential $\mathbf{A}=x y \mathbf{k}$, where $\mathbf{k}$ is a unit vector along the $z$ axis. For such a field, $\mathbf{B}=(x,-y, 0)$, and the motion of a charged particle in this field is governed by the reduced Hamiltonian (Jaroensutasinee and Rowlands, 1994)

$$
H=\frac{1}{2}\left[\dot{x}^{2}+\dot{y}^{2}+(Q-x y)^{2}\right],
$$

where $Q$ is a constant proportional to the $z$ component of the momentum and $\dot{x} \equiv d x / d t$. An immediate consequence of the constancy of $H$ (which in the following we normalize to $\frac{1}{2}$ ) is that the particle motion is confined to regions between the curves $y=(Q \pm 1) / x$. Thus, for $Q>1$, the particle is excluded from the origin, the position of the zero of the magnetic field. For $Q \gg 1$, adiabatic theory applies, and the value of the jump $\Delta \mu$ was given some time ago by Howard (1971). A typical orbit is shown in Fig. 1(d).

For $Q<1$, the origin is no longer excluded, and particle orbits may pass through or close to the zero-magnetic-field region. Some typical orbits are shown in Fig. 1. A subset of these orbits (Figs 1a, b) are such that they remain close to the $x$ axis, and it is for this type of orbit that we now develop a novel analytical approach. The case $Q=0$ is developed in detail, although the method is applicable to all $Q<1$.

The exact equations of motion are simply

$$
\frac{d^{2} x}{d t^{2}}=-x y^{2}, \quad \frac{d^{2} y}{d t^{2}}=-x^{2} y,
$$

whilst the adiabatic invariant $\mu$ (the ratio of the perpendicular kinetic energy to the magnitude of the magnetic field) is given by

$$
\mu=\frac{1}{\left(x^{2}+y^{2}\right)^{3 / 2}}\left[(\dot{x} x+\dot{y} y)^{2}+x^{2} y^{2}\left(x^{2}+y^{2}\right)\right] .
$$



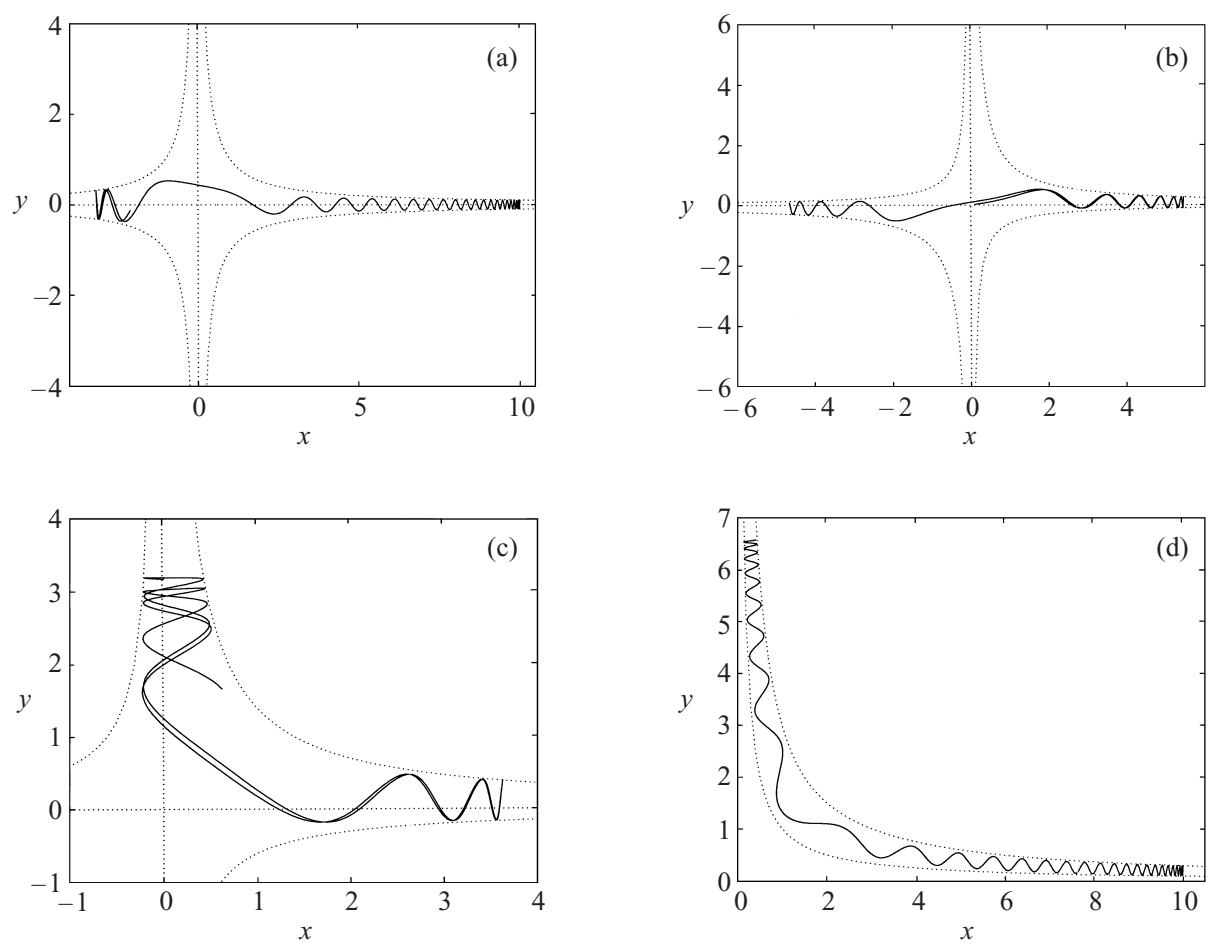

Figure 1. Typical orbits in the linear cusp field: (a) $Q=0$; (b) 0.4 ; (c) 0.4 ; (d) Dotted lines are $y=(Q \pm 1) / x$.

For the type of orbit under discussion, we write $y=g(x)$ and take $g$ to be a monotone function of $x$. Then, using the equations of motion, we find that $g$ satisfies the following differential equation:

$$
\frac{d^{2} g}{d x^{2}}=\frac{1}{1-x^{2} g^{2}}\left[x g\left(1+g^{\prime 2}\right)\left(g g^{\prime}-x\right)\right]
$$

where $g^{\prime}=d g / d x$.

It is apparent from Figs $1(a, b)$ that the orbits that pass close to the region where the magnetic field is zero are such that the value of $y$ is small for a range of values of $x$. Thus we treat both $g$ and $x$ as small, and linearize the above equation in $g$ about $x=0$ to give $d^{2} g / d x^{2}=-x^{2} g$, whose solution is

$$
y=A \sqrt{x} J_{1 / 4}\left(\frac{1}{2} x^{2}\right)+B \sqrt{x} J_{-1 / 4}\left(\frac{1}{2} x^{2}\right),
$$

where $A$ and $B$ are constants and $J_{ \pm 1 / 4}$ are Bessel functions (Nip et al. 1994). However, such a solution for all $x$ is totally inappropriate, since it excludes the possibility of the particle being reflected in regions of high magnetic field (large $x$ ), which is clearly the case as shown in Fig. 1. On the other hand, it is in such regions that adiabatic theory is valid. Thus, in the following, we obtain an approximate analytical expression for the whole orbit of a particle by combining the above form, valid for small $x$, with an expression, valid for large $x$, obtained by assuming constancy of the adiabatic invariant.

With $y=g(x)$, so that $\dot{y}=g^{\prime}(x) \dot{x}$, one can use the expression for the Hamiltonian given by (1) to obtain an expression for $\dot{x}$ and $\dot{y}$ in terms of $x, y$ and 
$g^{\prime}(x)$. This is then substituted into the expression for $\mu$, as given by (4), to give the exact relation

$$
\left(\mu-x g^{2}\right) x^{3}=\frac{\left(1-x^{2} g^{2}\right)\left(g+x g^{\prime}\right)^{2}}{1+g^{\prime 2}} .
$$

The turning point of a trajectory $(x=\bar{x})$ is where $g=0$ and $g^{\prime}=\infty$. The above equation gives $\mu \bar{x}=1$, so the turning point is uniquely specified by the value of $\mu$. If $x_{n}$ is the position of the $n$th zero of $g$, the above equation can be rearranged to give

$$
g^{\prime 2}\left(x_{n}\right)=\frac{x_{n}}{\bar{x}-x_{n}} .
$$

On the other hand, if $x_{m}$ is the position of $m$ th zero of $g^{\prime}(x)$, then, neglecting 1 and $g\left(x_{m}\right)$ compared with $x_{m}$, (7) reduces to

$$
g\left(x_{m}\right)=\sqrt{\frac{\mu}{x_{m}}} .
$$

This is a good approximation because, as seen from Fig. 1, the particles move deep into the regions where the magnetic field is large where $y$ is small.

We now use these values of $g$ and $g^{\prime}$, obtained at specific points, to fit $y(x)$ for all large values of $x$ to a form suggested by the numerical results shown in Fig. 1 , namely

$$
y(x)=D(x) \cos \left[\int_{0}^{x} \lambda\left(x^{\prime}\right) d x^{\prime}+\phi\right],
$$

where $D(x)$ and $\lambda$ are to be taken as slowly varying functions of $x$. To obtain expressions for $D(x)$ and $\lambda(x)$, we equate the value of $y^{\prime}(x)$ calculated at values of $x^{\prime}$ where $y(x)=0$, namely $\lambda(x) D(x)$, with the value obtained above at the discrete values $x_{n}$. Thus

$$
[\lambda(x) D(x)]^{2}=\frac{x}{\bar{x}-x} .
$$

Similarly, at the zeros of $y^{\prime}(x)$, we obtain the relation $D(x)=\sqrt{\mu(x)}$, where in this case we have neglected terms of order $1 / x^{3 / 2}$. Thus

$$
\lambda(x)=\sqrt{\mu(\bar{x}-x)} .
$$

We now have an explicit solution for $y(x)$ in terms of the two constants $\mu$ and $\phi$. To obtain a solution for all $x$, we combine the features of the solution for small $x$, as given by (6), with that for large $x$, as given by (8), and propose a solution for all $x$ of the form

where

$$
y \equiv g(x)=A \sqrt{\frac{2 h(x)}{x}} J_{1 / 4}(h(x))+B \sqrt{\frac{2 h(x)}{x}} J_{-1 / 4}(h(x)),
$$

$$
h(x)=\int_{0}^{x} d x \lambda(x)=\frac{2\left[2 \bar{x}^{3 / 2}-2(2 \bar{x}+x) \sqrt{\bar{x}+x}\right]}{3 \sqrt{\mu}} .
$$

For small $x$, where $h(x)=\frac{1}{2} x^{2}$, this reduces to (4), whilst comparison of this form in the asymptotic region where $x$ and $h(x)$ are much larger than unity, with (8), gives

$$
A=-\sqrt{\frac{1}{2} \pi \mu} \sin \left(\phi+\frac{1}{8} \pi\right), \quad B=\sqrt{\frac{1}{2} \pi \mu} \cos \left(\phi-\frac{1}{8} \pi\right) .
$$



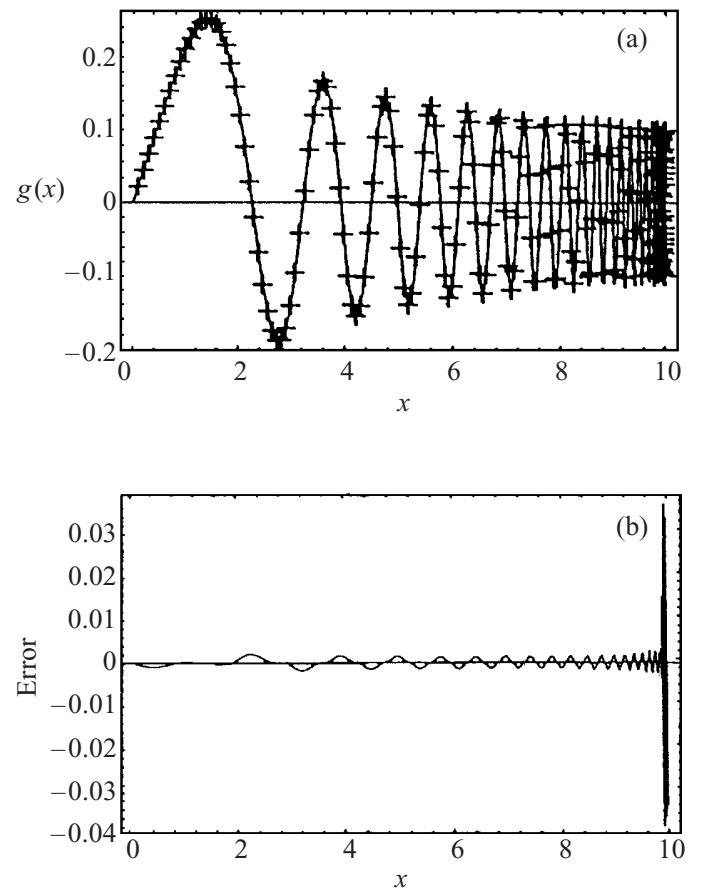

Figure 2. (a) Comparison of a numerically generated orbit with the analytic one. The crosses '+' indicate numerical results, while the solid line shows the analytical results. (b) Relative error of the analytical orbit from the numerically obtained one.

These last two equations can be rearranged to give

$$
A^{2}+B^{2}+\sqrt{2} A B=\frac{\pi \mu}{4} \equiv \frac{\pi}{4 \bar{x}}
$$

and

$$
\tan \phi=-\frac{B / A+a}{1+(B / A) a}
$$

where $a=\tan \left(\frac{3}{8} \pi\right)$. Thus the analytical expression for the orbit, as given by (9), agrees with adiabatic theory for large $x$ and the exact solution in the region of small $x$ and small $y$. The quantities $A$ and $B$ are obtained from initial conditions', as expressed for example by the values of $y$ and $\dot{y}(t)$ at $x=0$, whilst the value for $\mu$ (or $\bar{x}$ ) is obtained in terms of $A$ and $B$ using (11). The form for $y$, as given by (9), has similar features to a WKB solution, but here the solution is of a nonlinear problem.

The equations of motion for $x$ and $y$ have been integrated numerically, using a symplectic integrating routine (Rowlands 1991), starting at $t=0$ with $x=$ $y=0, \dot{x}(0)=p$ and $\dot{y}(0)^{2}=1-p^{2}$. Such an orbit corresponds to the case

$$
B=0, \quad A^{2}=2 \Gamma\left(\frac{5}{4}\right)^{2} \frac{1-p^{2}}{p^{2}}, \quad \phi=-\frac{3}{8} \pi, \quad \mu=\frac{1}{\bar{x}}=\frac{4 A^{2}}{\pi} .
$$

The integrator conserved energy to one part in $10^{-6}$. An orbit so obtained is shown in Fig. 2, where it is also compared with an orbit obtained from the 
analytical expression given by (9). The value of $p$ is chosen to give a value of $\bar{x}=10$, and it is this value that is used in (10) to uniquely determine $h(x)$. The agreement is surprisingly good over the whole range of possible values of $x$.

Equation (9) specifies an orbit that starts from $x=0$ in terms of the two constants $A$ and $B$. Eventually, the particle is reflected at $x=\bar{x}$, and then continues until it returns to the $x=0$ plane, but in general with different values of $y(0)$ and $\dot{y}(0)$. Importantly, the same equation (7) describes this return orbit, but now specified by different values of $A$ and $B$, which we denote by $\hat{A}$ and $\hat{B}$. These new values can be related to $A$ and $B$ simply by imposing continuity on the particle orbit. Thus we demand that both orbits give rise to the same value for $y$ when $x=\bar{x}$ and a value for $\dot{y}(t)$ equal in magnitude, but opposite in sign, corresponding to a reflection at $x=\bar{x}$. These conditions lead to a phase change in $\phi$ in the expression for $y$ as given by (8) such that the new value $\tilde{\phi}=$ $2 \pi-2 h(\bar{x})-\phi$. Since the turning point of both orbits is the same, both orbits have the same value of $\mu$. The adiabatic invariant $\mu$ does not change at the reflection point - only the phase does. Using (11) and (12), it is now possible to relate $(\hat{A}, \hat{B})$ and $(A, B)$, and we find

and

$$
\frac{\pi}{4 \bar{x}}=A^{2}+B^{2}+\sqrt{2} A B=\hat{A}^{2}+\hat{B}^{2}+\sqrt{2} \hat{A} \hat{B}
$$

$$
\sqrt{2}\left(\frac{\hat{B}}{\hat{A}}+\frac{B}{A}\right)+(1+b)=-\frac{\hat{B}}{\hat{A}} \frac{B}{A}(1-b),
$$

where $b=\tan \left(\frac{8}{3} \bar{x}^{2}\right)$.

The orbit illustrated in Fig. 2(a) has been followed numerically until it returned to the $x=0$ plane. With initial conditions corresponding to $A=0.2802$ and $B=0$, the numerically obtained solution gave values of $\hat{A}=0.3848$ and $\hat{B}=0.1670$ as the particle passed through the $x=0$ plane after one reflection at its mirror point $x=\bar{x}$ and of the order of 20 Larmor orbits. The corresponding values obtained using (9) are 0.3697 and 0.1604 respectively. This excellent agreement has been obtained without introducing any scaling parameters but merely demanding that both orbits have the same initial conditions. This same agreement has been found for a range of different orbits, showing that (9) gives an analytical expression which captures all the main features of the true orbit.

The particle now labelled by $(\hat{A}, \hat{B})$ continues to move, and enters the negative half $x$ space - but this is exactly equivalent to entering the positive half $x$ space with a simple change in the direction of the $x$ component of the velocity. The particle may now be considered as moving to the right in positive $x$ space, but labelled by $(-\hat{A}, \hat{B})$.

Thus we can specify the notion of a particle including many crossings of the $x=0$ plane by a map relating $A, B$ to $(-\hat{A}, \hat{B})$. This map is essentially given by (13) and (14). However, it can be simplified by considering particles moving through the $x=$ plane in the positive direction only. Thus if $\left(A_{n}, B_{n}\right)$ labels the $n$th such crossing, we find

$$
\begin{gathered}
A_{n+1}=-\sqrt{2} A_{n} \cos h_{n}+B_{n}\left(\sin h_{n}-\cos h_{n}\right), \\
B_{n+1}=A_{n}\left(\cos h_{n}+\sin h_{n}\right)-\sqrt{2} B_{n} \cos h_{n},
\end{gathered}
$$

where $h_{n}=\frac{8}{3} \bar{x}_{n}^{2}$ and depends on $\left(A_{n}, B_{n}\right)$ as expressed by (13). One can use these 
equations together with (11) and (12) to calculate $\mu_{n+1}$ in terms of $\mu_{n}$ and the phase $\phi_{n}$. To this extent, it is a generalization of the Chirikov map to the case where the particle passes in the vicinity of a null point in the magnetic field. However, it is limited to orbits that always remain in the ' $x$ ' arms (or, by symmetry, the ' $y$ ' arms) of the field. Such orbits are shown in Figs 1(a, b).

This map has been studied numerically, and shows chaotic behaviour for most initial conditions. It is found that the value of $\mu$ gradually increases owing to chaotic diffusion, which means that $\bar{x}$ decreases and the particle is reflected at a smaller value of $x$. This can lead to the particle moving into an arm of the cusp at right-angles to its present one. An example of such an orbit is shown in Fig. 1(c). However, once it is in the ' $y$ ' arm, it moves initially such that $x$ is small and then into an adiabatic region with $y$ large. Thus the analytical form of the orbit can still be described by (9), but with $x$ and $y$ interchanged. We specify this orbit by $(\tilde{A}, \tilde{B})$. Now a particle specified by $(A, B)$ undergoing one reflection in an $x$ arm, returns to the vicinity of the origin, and is specified by $(\hat{A}, \hat{B})$ as discussed above. For small $x$, one can use $(9)$ to express this orbit in the form $y=\alpha_{1} \hat{B}+\alpha_{2} \hat{A} x$, where $\alpha_{1}=\sqrt{2} / \Gamma\left(\frac{3}{4}\right)$ and $\alpha_{2}=1 / \sqrt{2} \Gamma\left(\frac{5}{4}\right)$. This same orbit, expressed as one that eventually moves in a ' $y$ ', arm now takes the form $x=\alpha_{1} \tilde{B}+\alpha_{2} \tilde{A} y$. Simple algebra then shows that $\tilde{A}=1 / \alpha_{2}^{2} \hat{A}$ and $\tilde{B}=-\hat{B} / \alpha_{2} \hat{A}$. Thus we can express the initial conditions for a particle moving into a ' $y$ ' arm, namely $(\tilde{A}, \tilde{B})$, in terms of $(\hat{A}, \hat{B})$ and hence $(A, B)$, which specify the previous excursion in a ' $x$ ' arm. Finally noting that a particle will only switch from an ' $x$ ' arm to a ' $y$ ' arm if the slope of the orbit near $x=0$ is greater than unity, that is $\alpha_{2} \tilde{A}>1$, one can construct a map that generalizes that given above to allow for transfer between arms. This takes the form

$$
\left(A_{n+1}, B_{n+1}\right)=\left\{\begin{array}{lll}
\left(-\hat{A}_{n} \hat{B}_{n}\right) & \text { if } & \left|\alpha_{2} \hat{A}_{n}\right|<1, \\
\left(\frac{1}{\alpha_{2}^{2} \hat{A}_{n}},-\frac{\hat{B}_{n}}{\alpha_{2} \hat{A}_{n}}\right) & \text { if } & \left|\alpha_{2} \hat{A}_{n}\right|>1 .
\end{array}\right.
$$

This map is a generalisation of the Chirikov map to allow a particle to move in regions where the magnetic field is small, and even zero, and the concept of an adiabatic invariant, such as $\mu$, which can have small jumps $\Delta \mu$, is no longer valid.

A preliminary numerical investigation has been made using this map. It is found that particles are contained in a finite portion of the relevant phase space, that is with finite values of $A_{n}$ and $B_{n}$, reflecting the boundedness of exact particle orbits due to energy conservation. The phase-space plots of typical orbits show little structure, implying that the particle motion is chaotic for most initial conditions.

\section{Conclusions}

An approximate but analytical expression has been obtained describing orbits of charged particles moving in the neighbourhood of a null point in a magnetic field. Comparison with direct numerical evaluation of particle orbits show that this analytical form is surprisingly accurate. Typical orbits have been followed over a time interval that includes many Larmor oscillations, with a resulting accuracy of a few percent. 
This analytical form has been used to obtain a map that specifies an orbit in the vicinity of the magnetic field null in terms of two quantities $\left(A_{n+1}, B_{n+1}\right)$, and relates these to the previous values $\left(A_{n}, B_{n}\right)$ when the particle was in the same region. This map can be used to study the long-time behaviour of particles (many crossings of the null region) more efficiently in computer time than a direct numerical integration of the particle-orbit equations. Preliminary results suggest that this map shows chaotic behaviour for all or most initial conditions.

Acknowledgement

We wish to thank the Development and Promotion of Science and Technology Talents Project (DPST) of the Thai government for financial support for one of us (K. Jaroensutasinee).

\section{References}

Cohen, R., Rowlands, G. and Foote, J. H. 1978 Phys. Fluids 21, 627.

Howard, J. E. 1971 Phys. Fluids 14, 2373.

Jaroensutasinee, K. and Rowlands, G. 1994 J. Phys. A27, 1163.

Lichtenberg, A. J. and Lieberman, M. A. 1983 Regular and Stochastic Motion. SpringerVerlag, New York.

Nip, M. L. A., Tuszynski, J. A., Otwinowski, M. and Dixon, J. M. 1992 J. Phys. A25, 5553. Rowlands, G. 1991 J. Comput. Phys. 97, 235. 